\title{
eJRIEPS
}

Ejournal de la recherche sur l'intervention en éducation physique et sport

15 | 2008

Varia

\section{Technologie des APSA : évolution des recherches et de leur place dans le cursus STAPS}

\section{Daniel Bouthier}

\section{OpenEdition}

\section{Journals}

Édition électronique

URL : http://journals.openedition.org/ejrieps/5831

DOI : 10.4000/ejrieps.5831

ISSN : 2105-0821

Éditeur

ELLIADD

\section{Référence électronique}

Daniel Bouthier, «Technologie des APSA : évolution des recherches et de leur place dans le cursus STAPS », eJRIEPS [En ligne], 15 | 2008, mis en ligne le 01 juillet 2008, consulté le 02 mai 2021. URL http://journals.openedition.org/ejrieps/5831 ; DOI : https://doi.org/10.4000/ejrieps.5831

\section{(c) (1)}

La revue eJRIEPS est mise à disposition selon les termes de la Creative Commons Attribution 4.0 International License. 
Technologie des APSA : évolution des recherches et de leur place dans le cursus

STAPS

Daniel Bouthier

IUFM d'Aquitaine, Mérignac cedex, France

LACES EA 4140, Universités Bordeaux 2-Bordeaux IV

Résumé

Haudricourt (1964) considère la technologie comme une science humaine des techniques. En APSA, la réhabilitation des techniques corporelles, après la période critique du technicisme, date du début des années quatre-vingt (Garassino, 1980 ; Vigarello, Vives, 1985). Qu'en est-il aujourd'hui des recherches en technologie en APSA et quelle place occupent-elles en STAPS dans les formations? La vision enrichie des techniques et l'émergence de la technologie, comme approche rationnelle de la genèse et de la transmission des techniques, ont permis de passer d'une vision essentiellement "technocentrée " à une approche "anthropo-techno-didactique ». Notamment sous l'impulsion d'Alain Durey, ces quinze dernières années ont vu la production régulière de thèses en technologie des APSA qui permettent aujourd'hui un bilan contrasté. Le volume de recherche dans le domaine n'est plus insignifiant et des outils conceptuels et méthodologiques de production de connaissances technologiques répondent à des critères de rigueur et des normes de crédibilité. Cependant ils sont peu valorisés en STAPS et rarement mis à disposition dans les formations initiales et continues en EPS.

Dans le cadre des 1ères Journées Alain Durey, d'actualité de la recherche en Didactique et Technologie des APSA, il est nécessaire de resituer le contexte de l'un des creusets de développement de cette perspective d'investigation. Alain est nommé à l'UFR des Sciences d'Orsay de l'Université Paris-Sud 11, à la fin des années quatre-vingt, avec Jean-Louis Martinand et Philippe Varin, suite au déplacement de l'ENS St Cloud à Lyon. Nous sollicitons son affectation à la Division STAPS, qui est acceptée par l'Université. La Division STAPS est à l'époque une jeune structure associant le cursus STAPS et la vie sportive de l'Université. Elle regroupe une bande de "doux dingues" (Blais, Bouthier, Cleuziou, David, Dumontaux, Legein, Lombardo, Papelier, etc.) qui visent à mettre à la 
portée du plus grand nombre la culture physique sportive et artistique, à partir d'une expérience corporelle riche, partagée et explicitée. Configuration prometteuse et visée ambitieuse au sein de laquelle ce physicien va pouvoir jouer le rôle d'accélérateur de particules dans la «Chaufferie » désaffectée, qui sert de laboratoire. S'en suit une densification du collectif de recherche (enrichie aussi du savoir-faire et de la disponibilité à toute épreuve de Philippe Varin); la création de l'option de «Didactique des APS » (Bouthier, Durey, Marsenach) du DEA parisien de Didactique des Disciplines; la stimulation des recherches en Technologie des APSA. Personnellement, je retiens le bonheur d'avoir été «en prise directe » avec Alain, sur la base d'une expérience comparable ou partagée : sensible (engagement et respect d'autrui), motrice (complicité d'anciens rugbymen), cognitive (idéaux humanistes, visées techno-didactiques), sociales (DEA, ACAPS-ARIS, SNESUP-SNEP, CNU). Alain Durey, c'est donc le souvenir de l'élégance du cœur et de l'esprit...

\section{Le contexte du développement d'une perspective technologique en STAPS}

Ce contexte est à considérer à la fois du point de vue institutionnel et scientifique.

\section{1. Le contexte institutionnel et organisationnel}

II est en évolution rapide. 1978 voit passer la dernière promotion recevant une formation professionnelle en EPS issue des classes préparatoires, des IREPS et CREPS. Les cursus STAPS sont complétés par des maîtrises en 1981-82 et les concours de recrutement s'enrichissent de l'agrégation externe d'EPS, en 1983-84. Dans les années quatre-vingt-dix, les UEREPS sont remplacés par des UFRAPS, qui ne prennent plus seulement en charge la préparation aux concours EPS, mais tout le spectre des métiers du sport (en concurrence avec le Ministère de la Jeunesse et des Sports). Chez la plupart des enseignants en STAPS, mais ce n'est sans doute pas seulement le cas en STAPS, les sciences sont conçues comme un réservoir de connaissances, en surplomb des pratiques. Ces dernières se réduiraient en outre à une application de techniques qui pourraient elles mêmes être mécaniquement déduites des sciences. Ainsi comme nous le constations récemment (Bouthier, 2006), les STAPS sont le plus souvent fonctionnellement segmentées d'une part en sciences appliquées aux APS (SAAPS) et en techniques des APS (TAPS) enseignées sous forme de théorie et pratique. Malgré tout un certain nombre de travaux prennent les pratiques corporelles comme objet spécifique ayant son épaisseur et donnant lieu à des investigations propres. Par contre, peu d'entre eux relèvent de la Technologie comprise comme "science humaine des techniques » (Haudricourt, 1987) pour formaliser les techniques des pratiquants et des intervenants en 
APSA (Bouthier, Durey, 1994). Ainsi reste un enjeu important : développer des recherches pour connaître, comprendre et agir, donc produire des connaissances répondant aux besoins des secteurs sociaux de pratique corporelle et prenant les problèmes de terrain de ceux-ci comme objet central d'étude.

Aujourd'hui la plupart des UFRAPS ont transformé leur intitulé en "Faculté » (sigle antérieur aux UER et UFR et qui permet de s'attribuer le titre de «Doyen ») des «Sciences du Sport », au nom d'une lisibilité et d'une harmonisation internationale en partie fictive, puisque on trouve à l'étranger des sciences du sport mais aussi des sciences de l'éducation physique, de la kinésiologie, de la motricité humaine, etc. C'est d'ailleurs pour ce nouvel intitulé de "Motricité Humaine" que se prononce la majorité des directeurs de composantes STAPS au sein de la Conférence des Directeurs d'UFRAPS et départements STAPS (Journée thématique : Cabourg, 10 juin 2005), et les membres de la $74^{\mathrm{e}}$ section du Conseil National des Université (CNU, séance introductive de la session de qualification: 31 janvier- 4 février 2005). Dommage, alors que la population française reconnaissait enfin ce sigle des STAPS (suite à la densification de la filière et à ses luttes sociales), que certains en proposent l'abandon, en particulier du $\mathrm{T}$ (de technique et technologie) et des APS... comme pratiques de référence. Récemment, le double jeu de recomposition des masters et des laboratoires vient de faire disparaître, dans la plupart des cas, la référence explicite aux STAPS dans les intitulés. Ainsi, les cursus STAPS sont intégrés dans des structures de recherche, et des diplômes relatifs à la Motricité, au Vieillissement, à l'Ergonomie, à l'Education, etc. De fait, la nécessité de production de connaissances sur les pratiques physiques sportives et artistiques pour répondre aux besoins sociaux s'évanouit du paysage universitaire français dans sa formulation explicite.

\section{2. Le contexte théorique}

Pourtant des points d'appui théoriques existent avec les travaux historiques, ethnologiques, anthropologiques, philosophiques, sur les techniques, la technicité, la technologie (Simondon, 1958 ; Leroi-gourhan, 1964 ; Gille, 1978). Les techniques sont en effet, non plus seulement conçues comme la forme extérieure du geste du champion dans le technicisme, mais comme l'ensemble des procédures (mentales et motrices) socialement capitalisées et transmises. La technicité renvoie à une forme particulière de rationalité, mettant en synergie des éléments de connaissance, d'expérience et de l'environnement pour agir. Elle mobilise différents registres de lecture, reproduction et transformation des techniques existantes, et de participation aux productions sociotechniques, à travers la possibilité d'assumer différents rôles (Combarnous, 1984). La technologie, au-delà des réalisations matérielles sophistiquées, est considérée comme 
une science humaine de l'intelligence mise jeu dans la conception, la mise au point et l'évaluation de créations, une science de l'artificiel générant des artéfacts cognitifs, matériels, et bien sûr corporels. La réflexion philosophique et sociologique sur la technologie (Ministère de la recherche et de la technologie 1982; Feenberg, 2004) souligne le caractère social des choix technologiques qui sont souvent pluriels et doivent être démocratiquement tranchés selon des critères éthiques, écologiques, esthétiques. Enfin, le développement des travaux des technologues et des didacticiens de la technologie (Deforge, 1970, 1993; Rabardel, 1998; Martinand, 1986), étayent et amplifient ce mouvement, mettant en évidence l'intérêt d'une culture technique pour tous, et la nécessité de recontextualiser l'élaboration, la transmission et l'évolution des techniques. Enseigner des procédés et des procédures ne suffit pas, pour en apprécier l'efficience il faut en connaître le sens. II faut accéder à l'intentionnalité initiale, aux conditions d'élaboration (mentalités dominantes, connaissances disponibles, pratiques usuelles, outils disponibles), aux raisons d'être donc des techniques, qui sont datées, situées et appartiennent à des lignées techniques. Du fait de la reconnaissance progressive de l'importance d'une réflexion sur la technologie y compris en APS (Garassino, 1980 ; Vigarello, Vivés, 1985 ; Vigarello, 1988 ; Bouthier, 1993 ; Goirand, Metzler, 1996 ; Lafont, Bouthier, 2004 ; Legras, 2005 ; etc.), il semble essentiel de s'interroger sur la production actuelle de recherches technologiques en STAPS.

\section{Les recherches en technologie des PPSA en France}

Avant de caractériser les recherches en technologie des APSA, il convient de poser une première définition provisoire, de leur objet même.

2. 1. Les pratiques physiques sportives et artistiques (PPSA) comme objet d'investigation

Comme nous le remarquions (Bouthier, 1993), par Activités Physiques Sportives et Artistiques, la communauté des STAPS et de l'EPS désigne en fait des pratiques, au sens sociologique du terme. En effet ce sont bien des productions de groupes humains, repérables et institutionnalisées, qui sont évoquées par ce sigle. Celles-ci prennent des formes et donnent lieu à des usages divers, culturellement constitués et géographiquement situés. Elles sont composées, selon Martinand (1986), de valeurs, d'émotions, d'attitudes, de savoirs, de procédures, de rôles, etc. Elles reposent sur des interactions sociales, des expériences partagées, des échanges et des métissages. La notion d'activité renvoie, en psychologie, aux engagements individuels, aux motifs personnels d'agir, aux buts consciemment retenus par le sujet, aux opérations mises en 
œuvre dans son rapport subjectif, particulier au monde (Léontiev, 1976, 1984). Ainsi, il serait plus judicieux de dire, à l'inverse des habitudes professionnelles que "les élèves activent (au sens de donnent vie ici et maintenant) une pratique (qui existe par ailleurs en dehors d'eux) », plutôt qu'ils pratiquent une activité. En tout état de cause, c'est bien la prise en compte de ces deux plans, et ce double jeu de transmission / appropriation de modèles culturels inscrits dans les pratiques et d'individualisation de ces modèles culturels (re)créés par les individus en activité, qu'il est important de prendre en compte.

Les PPSA remplissent des fonctions sociales particulières. L'analyse anthropologique, historique et sociologique conduit à distinguer les différentes fonctions remplies par ces dernières selon les époques et les groupes humains, comme :

- se classer en établissant une performance ;

- contrôler les jeunes dans des organisations ;

- construire, préserver, rétablir la santé ;

- faire des profits financiers ;

- conserver des activités et des instruments culturels ;

- détourner l'attention de problèmes sociaux cruciaux ;

- Construire par l'éducation des rapports humains citoyens ;

- etc.

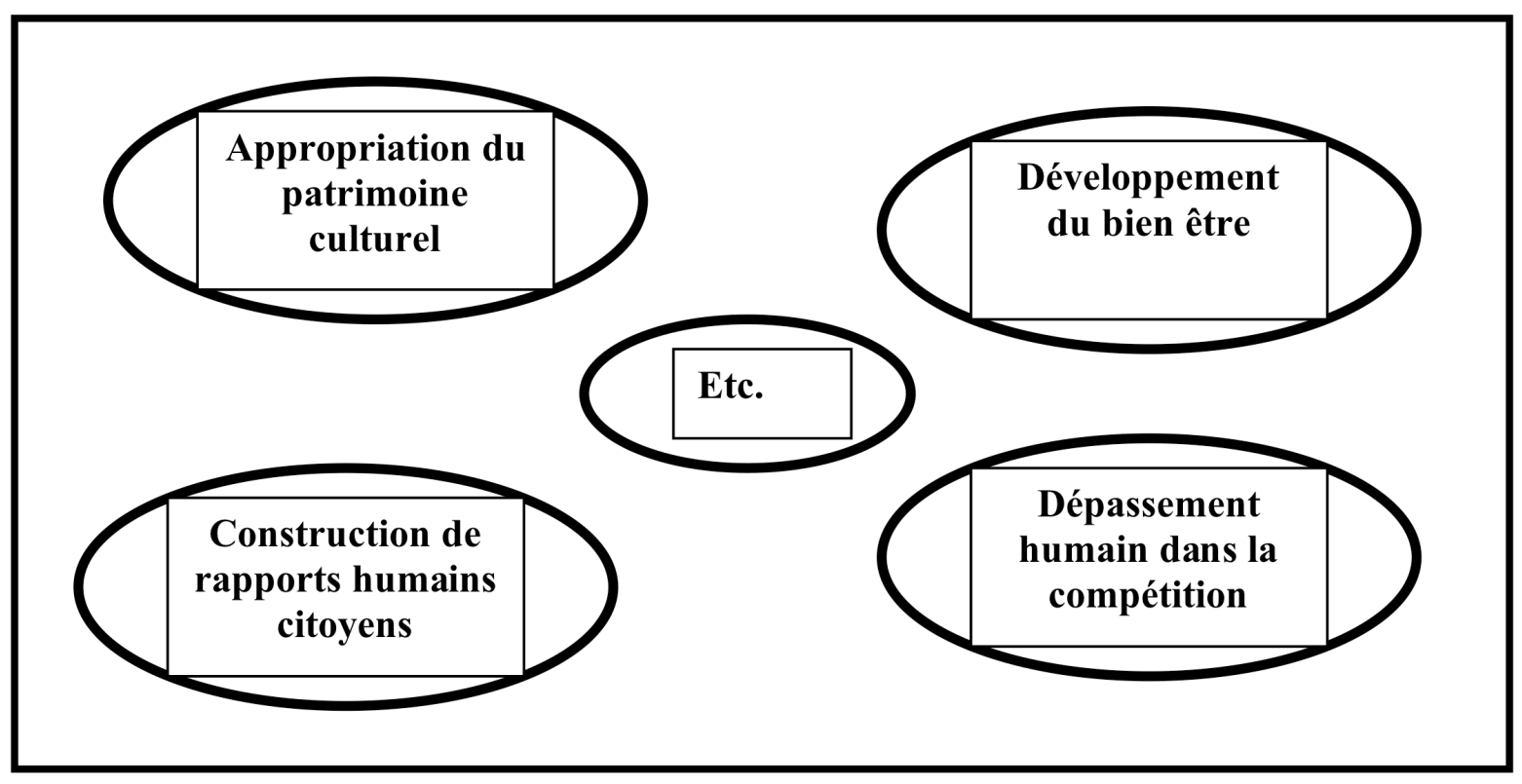

Figure 1. Fonctions sociales des PPSA heuristiques pour l'intervention éducative

Ces significations sociales sont souvent présentes, pour un certain nombre d'entre elles, avec des poids divers, au sein d'une même pratique d'un groupe humain. Elles constituent 
des unités contradictoires. Elles ne sont pas toutes recevables dans une perspective éducative.

II est donc important, dans notre domaine notamment, de ne pas considérer une PPSA comme univoque. A titre d'exemple, le basket constitue-t-il une entité homogène ? Les différences entre le basket de la NBA, le basket scolaire et le street-basket sont-elles négligeables ? Le basket peut-il être réduit au seul basket de haut niveau ? Et celui-ci doitil, seul, servir de modèle à tous les autres niveaux et types de pratique ? La notion de pratique sociale, nous alerte sur le fait que celles-ci sont plurielles. Mais la notion de référence n'induit pas comme seule opération possible la reproduction. Donc quel(s) basket(s) prendre pour référence pour telle pratique éducative en EPS ? Et la référence renvoie-t-elle à l'idée de prendre cette pratique pour cible, à terme de la reproduire, de la dépasser, de la transformer, de la repousser (ce qui est aussi y faire référence par la négation) ? Encore faut-il que les pratiques soient reconnues comme des objets dignes d'investigations.

\section{2. Caractérisation des recherches en technologie des APS}

Si l'on prend comme indicateur la création des Maîtrises STAPS en 1981-82, qui lance institutionnellement la production dans la filière de connaissances spécifiques ancrées sur des recherches, on peut schématiquement distinguer deux périodes :

La première période (1982-1992) correspond à une vision encore très technocentrée et une production discontinue de faible volume. Ces recherches ne se réclament pas d'une perspective explicitement technologique, disqualifiée par une conception dominante étriquée et de ce fait très critique des techniques. Pour les enseignants-chercheurs en STAPS, souvent en recherche de rupture avec le monde professionnel, les pratiques (d'enseignement et d'entraînement) sont perçues comme péjoratives. L'objet des STAPS est mal délimité, sans paradigme spécifique affirmé. Il est donc plus rapide et confortable de s'installer dans des cadres conceptuels et méthodologiques de sciences d'appui déjà rodés. Si l'intérêt d'une perspective technologique est envisageable et potentiellement jugée pertinente, comme le plaide notamment Vigarello, elle reste à encore à construire.

La deuxième période (de 1992 à nos jours) voit se dégager une approche que nous pourrions qualifier d' " anthropo-techno-didactique ». En effet les techniques sont alors recontextualisées (origines, environnement, finalités), conçues comme évolutives sous les influences de leurs migrations géographiques et d'usages liées aux interactions humaines, et étudiées comme un contenu culturel orienté vers la transmission. Si le volume de production reste limité, il devient plus continu puisque l'on peut évaluer le régime de croisière actuel de deux à quatre thèses en technologie des APSA par an. Cette 
perspective est aujourd'hui admissible dans la communauté STAPS, dans la mesure où elle donne lieu régulièrement à la qualification des auteurs de ces travaux aux fonctions d'enseignant-chercheur. II est à noter que ce sont principalement la didactique et l'histoire, qui servent de niche écologique en abritant et stimulant ces types de recherches. Les recherches récentes en technologies des PPSA permettent d'une part de conceptualiser l'unité contradictoire entre sens et efficience, les registres de technicité étant mis en perspective au regard de l'intentionnalité de l'action et du patrimoine de la culture technique. Et d'autre part, elles autorisent une remise en perspective des rapports réciproques entre pratiques professionnelles et activités de formation, notamment à travers l'étude des savoirs utiles pour l'action, des expériences favorables à leur reconstruction et des compétences techniques ainsi développées (cf. figure 2).

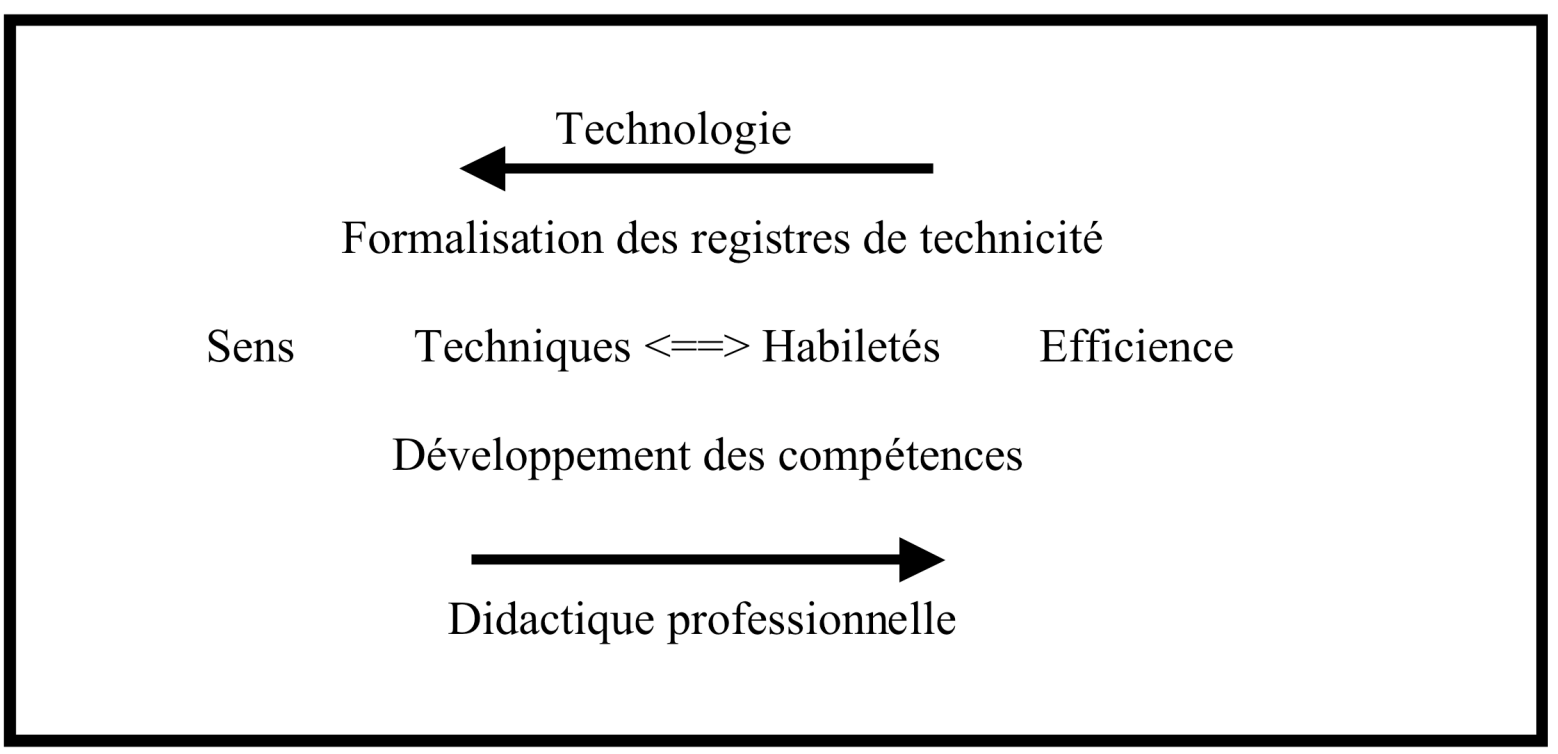

Figure 2. Symbiose technologie des PPSA et didactique de l'EPS

D'après Bouthier, D (2000). Symposium « didactique », Grenoble, ARIS, CD-Rom .

Les connaissances produites portent notamment sur:

- Les significations sociales des pratiques, les finalités visées, les fonctions remplies ;

- La spécificité, l'essence des pratiques, les valeurs sous-jacentes privilégiées ;

- Les conventions centrales de jeu (codes, règles), qui matérialisent la spécificité et les conventions secondaires, qui prennent du sens avec les différents niveaux et contextes de pratique ;

- Les logiques de comportements, qui renvoient à des stratégies d'organisation des actions au regard des exigences règlementaires et des conditions de pratique ;

- Les principes de mise en œuvre des opérations décisionnelles, motrices, émotionnelles, relationnelles en jeu dans les actions sportives et artistiques ; 
- Les compétences (en tant que connaissances procédurales et expériences partagées) et la technicité mise en jeu ;

- La conception et l'instrumentalisation d'outils techniques de lecture, de mesure, de simulation, de comparaison, des techniques corporelles et des techniques d'intervention en APSA ;

- Les conditions didactiques de la diffusion de la culture technique en APSA (s'éprouver corporellement, conscientiser les éléments cruciaux d'orientation, co-construire la mise en rapports des contextes typiques et des procédures pertinentes, etc.).

Ainsi il est possible de résumer les contenus et les médiations didactiques en usage dans l'intervention en éducation physique et en sport par la figure suivante.

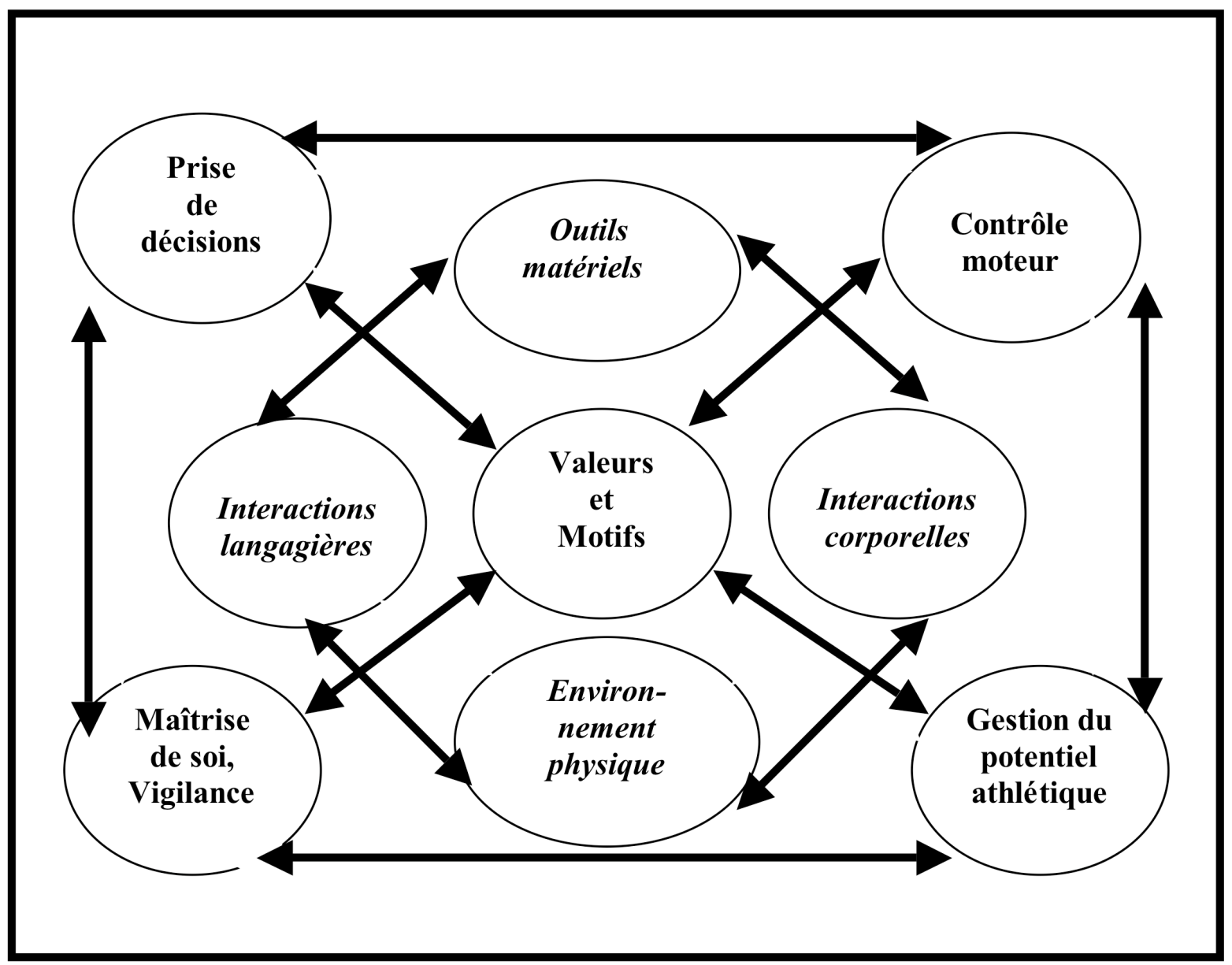

Figure 3. Contenus et médiations didactiques en EPS

Les contenus et les médiations didactiques formalisent le réservoir de pratiques de référence correspondant à une motricité ludique, sportive et artistique, dans des environnements physiques et humains inhabituels, reposant sur des valeurs (savoir vivre), des attitudes (savoir être), des connaissances (savoir), des procédures et des rôles (savoir faire), des expériences à vivre (savoir partager), etc. 
C'est l'étude de ces formalisations, leurs origines, les problèmes qu'elles visent à résoudre, les solutions qu'elles proposent, les évolutions qu'elles subissent, qui constituent le terreau des savoirs technologiques (savoirs sur la dynamique des techniques). Ces savoirs correspondent à des co-constructions composites évolutives qui amalgament des concepts scientifiques, qui peuvent être adaptés, obsolètes ou erronés, des savoirs professionnels (gestes du métiers, expérimentés, capitalisés et transmis), des intuitions et des expériences personnelles vécues et intégrées dans l'agir professionnel. La masse critique de travaux relevant de la perspective technologique permet aujourd'hui un retour sur les propositions méthodologiques faites à la charnière des deux périodes considérées (Bouthier, Durey, 1994).

2. 3. Méthodologie et critères de rigueur d'une recherche technologique en APSA :

Reprendrons ici l'exemple de la recherche relative au « Pocket Assistant Coach rugby », dans la mesure où elle a déjà été mise en perspective lors de précédentes journées d'études INRP-GéDIAPS en didactique des APS (Bouthier D., «Recherches en Technologie et didactique des APSA, et formation des enseignants en EPS », mars 2003, non publiée), et développée approfondie par $\mathrm{G}$. Uhlrich dans un article du même numéro de la revue. Cette recherche a été lancée au début des années 2000 à partir de questions cruciales de terrain :

- Doter les intervenants éducatifs (enseignants, éducateurs, entraîneurs) d'un outil d'analyse du jeu en temps réel pour l'aide au managérat des équipes de rugby en match (scolaires et fédérales) ;

- Répertorier les catégories (phases de mouvements, phases de fixation, phases statiques),

-Intégrer les opérations modernes d'analyse du jeu (conquête, récupération, utilisation, continuité) ;

- Matérialiser les représentations spatiales (espaces et phases de jeu défavorables) et temporelle (chaînes d'actions intra et interphases) du déroulement du jeu ;

- Comparer les équipes opposées par une approche quantitative et qualitative des actions ;

- Laisser à l'humain, la décision des consignes de jeu et des changements de joueur.

Ces visées pragmatiques renvoient à des problématiques scientifiques : développer et rendre accessible les instruments cognitifs et matériels de la technicité de lecture de la motricité (ici en sports d'opposition collective) de façon à armer les intervenants pour une évaluation diagnostique. Par un usage différé des données, il s'agit aussi de les aider à 
construire des évaluations formatives et sommatives des productions corporelles complexes.

A travers la focalisation sur un objet technique, ce processus de « recherche / développement » d'un produit logiciel innovant pour Pocket Digital Assistant (PDA) vise aussi la création d'un artéfact logiciel et la production de nouvelles connaissances technologiques et didactiques.

L'élaboration du cahier des charges de l'essai technique a nécessité :

- de faire l'état des connaissances sur les systèmes socio-techniques de production (activité des intervenants en compétitions sportives scolaires et fédérales) et de formation étudiés (formation des professeurs d'EPS et des Brevets d'Etat d'Educateurs Sportifs, à l'animation et au managérat des équipes) ;

- d'examiner les modélisations scientifiques possibles, les formalisations professionnelles disponibles, les intuitions personnelles tacites des concepteurs pour cerner les paramètres restant à préciser et à définir ;

- de mettre en œuvre les critères de rigueur spécifiques à ce type de recherche, notamment caractériser les points de vue adoptés, délimiter l'empan des systèmes étudiés, repérer les fonctions remplies et les leviers clés.

\section{3. 1. La conception}

Cette conception du projet «PAC rugby » s'est traduite par une description argumentée des fonctionnalités attendues de la base d'orientation sous-jacente, des conditions et des limites d'utilisation, qui ont permis d'organiser et de programmer les étapes du processus d'essai-évaluation technique, lui-même intégré dans le protocole de recherche technologique. Les critères de rigueur mis en jeu sont alors de l'ordre de la pertinence argumentée des emprunts en rapport avec le but visé par le projet, de la cohérence globale forte au regard des évolutions attendues par rapport à l'existant et d'un pronostic très favorable de faisabilité.

\section{3. 2. La mise au point}

La mise au point du produit s'est réalisée à travers de nombreuses séances de régulation entre concepteurs (commanditaires et programmeurs) et utilisateurs (managers d'équipe et formateurs d'enseignants et d'entraîneurs) avec une partie des membres ayant un statut mixte de concepteurs et d'utilisateurs. Ces séances ont permis l'affinement des éléments du projet, mais elles ont aussi provoqué des remaniements importants pour la forme et la viabilité du produit. Ces « négociations techniques » constituent un matériau crucial pour la recherche technologique en tant que lieux et moments clés de création de l'artéfact. 
Les critères de rigueur renvoient à l'expérience contrôlée, plus qu'à l'expérimentation programmée, des effets produits par la variation de paramètres du «modèle technologique prospectif élaboré » sur la réponse aux questions initiales de terrain.

\section{3. 3. L'évaluation}

L'évaluation du projet, suppose d'examiner les transformations permises, les connaissances produites, leurs conditions de diffusion. Le logiciel PAC rugby a maintenant une existence officielle. II est fonctionnel et commercialisé à un tarif le rendant accessible aux populations cibles visées (enseignants d'EPS et éducateurs de clubs amateurs). Mais il reste au début de son utilisation généralisée et de son évolution (extension à l'observation de l'activité du joueur et non seulement de l'équipe). Nous en sommes aux prémisses des résultats de la recherche technologique sur ce projet technique. Le PAC rugby produit des résultats et des représentations du jeu encore inhabituelles y compris pour les concepteurs. II va donc probablement générer à l'usage des évolutions des conceptualisations, des pratiques du managérat et de l'analyse du jeu.

Les critères de rigueur sont à ce niveau du domaine de la validité interne ou locale. Le projet est mené à son premier terme et répond aux attentes des commanditaires. II est utilisable pour manager des équipes en jeu et pour former de futurs enseignants et entraîneurs. Mais ces critères comportent aussi une exigence de validité externe, plus générale qui suppose sa diffusion professionnelle au-delà de son site initial de création. II s'agit de la divulgation et de la publication scientifique des résultats de la recherche technologique qui l'englobe. Les recherches technologiques supposent donc la constitution de collectifs pluricatégoriels associant, aux différentes étapes de celles-ci, chercheurs et praticiens.

\section{Recherches technologiques et cursus STAPS}

Les recherches technologiques devraient être constitutives du cœur des cursus STAPS. Pour autant, pour leur donner toute leur place et seulement leur place, il faut percevoir l'intérêt et les limites qu'elles présentent.

3. 1. Intérêt pour les cursus d'intégrer pleinement des résultats de recherches en technologie des PPSA

Alain Durey considère que les recherches en technologie des APSA ont un triple rôle à joueur dans les cursus STAPS (Durey, 1995) : diffuser en formation des résultats réinvestissables pour armer les néophytes de l'intervention éducative en sport, instrumentaliser en formation des outils de simulation de situations réelles (situations de diagnostic assisté, de micropédagogie, etc.), intégrer à la formation des professionnels de 
l'intervention éducative des problématiques et des méthodologies technologiques. Ces formations doivent donc fondamentalement porter sur des objets techniques (conceptuels, matériels, corporels) dynamiques, à évolution rapide, dans un contexte de conceptionessai-évaluation, de type expérience contrôlée, car elle-même en transformation au cours de sa réalisation. II s'agit donc de développer des compétences pré-professionnelles, dans un environnement de recherche - intervention, qui valorisent sans les sacraliser les connaissances scientifiques, les savoirs technologiques et les intuitions personnelles conçues comme dynamiques et interactives.

3. 2. Limites des perspectives scientifiques et techniques pour l'agir professionnel Les discours scientifiques et techniques disent à la fois plus et moins que la complexité du réel. Ils disent plus car ils ont en partie un caractère hypothétique et spéculatif par rapport au réel qu'ils dilatent et théorisent (Vigarello, Vivés, 1985). Ils en disent moins, car ils n'épuisent pas la complexité du réel. Ils laissent des zones d'ombre, d'incertitude sur les processus et les procédures en jeu dans certaines composantes de l'intervention (Staudenmaiër, 1988). Le chercheur peut avoir tendance à projeter sur l'action motrice d'un sujet des intentions et des opérations qui en sont éventuellement absentes. II peut aussi ignorer l'épaisseur de certains phénomènes que cela soit par absence de théorie correspondante ou du fait de l'incomplétude de l'explicitation des savoirs de l'agir. Ainsi, à la lumière du cognitivisme, ces vingt-cinq dernières années ont souvent conduit à focaliser sur les planifications de l'enseignement, minorant leurs adaptations ou remaniements en cours d'intervention. Certes elles sont susceptibles d'orienter, d'optimiser l'intervention sans pour autant pouvoir l'anticiper complètement, en particulier quand il est question d'interactions humaines. La revue de littérature scientifique et professionnelle réalisée par exemple sur le rugby (Bouthier, 2007) montre bien à la fois la largeur du spectre des angles d'approche scientifique utiles à la connaissance des mondes du rugby (anthropologie, histoire, sociologie, économie, technologie, didactique, physiologie, biomécanique, psychologie, etc.) et nécessaire à leur compréhension. Elles montrent en même temps leurs limites pour l'aide à l'intervention.

3. 3. Articulation et complémentarité des recherches, qui visent la production de connaissances et l'optimisation de l'intervention

Souvent en France, il est classique avec un petit caractère hiérarchique, de considérer d'une part la recherche dite fondamentale (qui créerait des connaissances nouvelles) et d'autre part, la recherche appliquée (de la précédente), qui en chercherait les développements possibles dans différents domaines potentiels d'application. Les travaux en histoire et en épistémologie des sciences et des techniques contestent cette vision 
(Staudenmaiër, op. cit.), qui occulte à la fois la part importante de transposition de remaniements (nécessaires pour ces recherches technologiques dites de développement) et la contribution qu'elle apporte souvent à des questions plus générales (Pestre, 1992). Ainsi en STAPS des travaux sur la formation aux prises de décision en sports collectifs, mettent en évidence le double registre décisionnel mobilisé en jeu prédictif ou planifié, et réactif ou régulé (Grehaigne, Godbout, Bouthier, 2000). Ils montrent que les joueurs ont des prévalences individuelles pour l'un des deux registres selon les conceptions du jeu et les formations reçues (Mouchet, Bouthier, 2006). Au-delà des sports collectifs, ils interpellent les tenants des approches cognitivistes, écologiques et énactives pour construire une vision plus articulée des différentes modalités d'initialisation et de contrôle des actions sportives complexes. Ils suggèrent, comme pour les filières bioénergétiques des transitions (ici dans l'action, entre fenêtres attentionnelles et émergences non intentionnelles) avec des possibilités de passage ultra rapides et réversibles. II en est de même pour les travaux réalisés, dans le cadre du programme collaboratif de la cellule recherche de rugby de la FFR, sur l'analyse des contraintes, la prévention et le traitement du rachis cervical en première ligne de mêlée dirigé par Portero et Gamet (contrat FFRMJS-GMF 2003-2007, collaboration Univ. Paris 12, UTC, CNAM, Institut de Myologie de France). A partir de l'analyse des forces de pression et des signaux électromyographiques, ils font apparaître l'inadéquation partielle du modèle en vigueur de la sidération de la commande musculaire à l'impact de l'entrée en mêlée pour inviter à examiner les perspectives offertes par un modèle de mise en résonance musculaire (Piscione, 2006). Cette nouvelle hypothèse, localement heuristique, invite à regarder en partie différemment les questions plus générales d'atteinte neuromusculaire (myopathies, etc.). Cette recherche, qui s'inscrit dans un programme à visée «fondamentale » sur les myopathies, ne débouche pas directement et immédiatement sur la production de connaissances nouvelles dans ce domaine mais elles génèrent des innovations technologiques majeures à propos de techniques de prévention (musculation et techniques de poussée), de contrôle (arbitres et joueurs) et de récupération (médecins et kinésithérapeutes) pour l'activité en première ligne de mêlées. Réciproquement les approfondissements, menés avec une visée initiale essentiellement "technologique », ouvrent de nouvelles perspectives pour ces questions scientifiques plus générales au plan de la myologie. 


\section{Conclusion}

Le développement des recherches technologiques en PPSA, la diffusion de leurs résultats dans les formations initiales en STAPS et professionnelles continues des enseignants, des éducateurs, des entraîneurs, etc., la familiarisation avec les objets techniques et la démarche technologique, constituent des enjeux majeurs pour les STAPS. Dans cette perspective il est utile de ne pas confondre à travers les STAPS : l'objet de la recherche, la discipline universitaire, et le cursus et les filières correspondantes. II est souhaitable en effet d'éviter la confusion du but, des conditions et des moyens. Pour autant, recherche et formation doivent être articulées, et s'enrichir mutuellement. De la même façon il est important de percevoir les différents statuts possibles de la discipline : discipline objet ou de cœur de cursus, discipline outil ou de service, discipline prospective ou d'ouverture (Martinand, 1992). II s'agit de réfléchir aux rôles, aux rapports entre disciplines et leurs formes de présentation (CM, TD, TP, stages, etc.) selon les filières du cursus et leurs niveaux. C'est donc un panorama contradictoire que l'on peut dresser des recherches technologiques en PPSA, qui sont à la fois en développement et en même temps parfois encore relativement marginalisées et dévalorisées en STAPS. II est toutefois légitime aujourd'hui au regard de leur masse critique, et décisif du fait de leur situation à l'interface des sciences et des techniques, de leur donner toute leur place dans les cursus STAPS. C'est en effet une des conditions pour que ces cursus prennent tout leur sens et se déploient dans toute leur portée.

\section{Références}

Bouthier, D. (1993). L'approche technologique en STAPS : représentations et actions en didactique des APS. Habilitation à Diriger des Recherches en Sciences, non publiée, Université Paris-Sud.

Bouthier, D. (2006). Conférence introductive à la table ronde « Sciences et techniques des APSA : quels rapports, vers quels métiers de l'intervention ? "Contre-pied, 18, CD-Rom.

Bouthier, D. (2007). Le rugby, Que-sais-je ? Paris : PUF.

Bouthier, D., \& Durey, A. (1994). Technologie des APS. Impulsions, 1, 95-124.

Combarnous, M. (1984). Comprendre les techniques et la technicité. Paris: Editions Sociales.

Deforge, Y. (1970). L'éducation technologique. Paris : Casterman.

Deforge, Y. (1993). De l'éducation technologique à la culture technique. Paris : ESF. 
Durey, A. (1995). L'EPS et son rapport à la technique. Spirales, 8, 93-95.

Feenberg, A. (2004). (Re)penser la technique. Paris : La découverte/MAUSS.

Garassino, R. (1980). La technique maudite. EPS, 164, 49-53.

Gille, B. (1978). Histoire des techniques. Paris : Gallimard.

Goirand P., Metzler J. (1996). Techniques sportives et culture scolaire. Paris : Laffont EPS.

Grehaigne, J-F., Godbout, P., \& Bouthier, D. (2001). The teaching and learning of decision making in team sports. Quest, 53, 59-76.

Haudricourt, G. A. (1987). Technologie science humaine. Paris : Maison des Sciences de l'Homme.

Lafont, L., \& Bouthier, D. (2004). Plaisir d'enseigner, désir d'apprendre: techniques corporelles et techniques d'intervention, enjeux et usages en didactique de l'EPS. In G Carlier (Ed .) Si on parlait du plaisir d'enseigner l'éducation physique (pp.8598). Montpellier : AFRAPS.

Legras, J.-M., (2005). Vers une technologie culturelle des APSA. Paris : Vigot.

Léontiev, A. (1976). Le développement du Psychisme. Paris : Editions Sociales.

Léontiev, A. (1984). Activité, conscience, personnalité. Moscou : Editions du Progrès.

Leroi-gourhan, A. (1964). Le geste et la parole: techniques et langages, t1. Paris: La Découverte.

Martinand, J.-L. (1986). Connaître et comprendre la matière. Berne : Peter Lang.

Ministère de la recherche et de la technologie (1982). Actes du Colloque National Recherche et Technologie. Paris, La Documentation Française.

Martinand, J-L. (1992). Organisation et mise en oeuvre des contenus d'enseignement. In J.Colomb (Ed.) Actes du colloque « Recherches en didactiques : contribution à la formation des maitres ». Paris, INRP.

Mouchet A., Bouthier D. (2006). Prendre en compte la subjectivité des joueurs de rugby pour optimiser l'intervention. STAPS, 72, 93-103.

Pestre, D. (1992). Les physiciens dans les sociétés occidentales de l'après-guerre. Revues d'Histoire Moderne et Contemporaine, 39(1), 54-72.

Piscione, J. (2006). Etude électromyogaphique des muscles striés squelettiques soumis à des compressions mécaniques externes: application à l'analyse de l'effort du joueur de première ligne en mêlée de rugby. Thèse de doctorat non publiée, Université Technologique de Compiègne.

Rabardel, P. (1995). Les hommes et les technologies. Paris : Armand Colin. 
Simondon, G. (1958, rééd. 1989). Du mode d'existence des objets techniques. Paris : Aubier-Montaigne.

Staudenmaiër, J. (1988). L'histoire des sciences et la question : les technologies sont-elles des ciences appliquées. Courrier du CETHES, 5, 27-43.

Vigarello, G. (1988). Une histoire culturelle du sport. Techniques d'hier et d'aujourd'hui. Paris : Laffont-EPS.

Vigarello, G., Vives, J. (1985). Technique corporelle et discours technique. Culture Technique, 13. 